\title{
Check-list das espécies de Fitoplâncton do estado do Mato Grosso do Sul
}

\author{
William Marcos da Silva' \& Fernanda Maria de Russo Godoy ${ }^{2}$
}

\author{
' Universidade Federal de Mato Grosso do Sul, Campus Pantanal, Av. Rio Branco 1270, Corumbá, Mato Grosso do Sul, Brasil. \\ wmsilvax@ig.com.br \\ ${ }_{2}^{2}$ Programa de Pós-Graduação em Biologia Vegetal, CCBS, Universidade Federal de Mato Grosso do Sul, Campo Grande, \\ Mato Grosso do Sul, Brasil. fergodoy_bio@hotmail.com
}

Recebido em 27.IX.2014

Aceito em 15.VI. 2016

DOI 10.21826/2446-8231201873s219

RESUMO - No estado de Mato Grosso do Sul foram registradas dez classes e contabilizadas 339 espécies fitoplanctônicas, destacando-se em riqueza de espécies as classes Chlorophyceae (66 espécies), Euglenophyceae (45 espécies), Cyanophyceae (34 espécies), Bacillariophyceae (25 espécies) e Zygnemaphyceae (16 espécies). Foram registradas 142 espécies na bacia do rio Paraná (42\%) e na bacia do Alto Paraguai foram registradas 197 espécies (58\%), com 55 espécies comuns entre as duas bacias (16\%). São discutidas as lacunas do conhecimento e as potencialidades dos estudos das comunidades fitoplanctônicas no estado.

Palavras-chave: água doce, bacia do Alto Paraguai, bacia do Paraná, microalgas.

ABSTRACT - Checklist of the Phytoplankton species from Mato Grosso do Sul state, Brazil. In the Mato Grosso do Sul state were recorded ten classes and 339 species, where the greatest richness were recorded in the Chlorophyceae (66 species), Euglenophyceae ( 45 species), Cyanophyceae (34 species), Bacillariophyceae (25 species) and Zygnemaphyceae (16 species). In the Paraná River basin were recorded 142 species (42\%) and in the Alto Paraguay River basin were recorded 197 species (58\%) and 55 species were common in the two Rivers basins (16\%). We discuss the knowledge gaps and potential studies of the phytoplankton communities in the state.

Keywords: Alto Paraguay River basin, freshwater, microalgae, Paraná River Basin.

\section{INTRODUÇÃO}

O fitoplâncton é um grupo taxonomicamente diverso, englobando quatro reinos: Bacteria, Protista, Chromista e Plantae (Cavalier-Smith 2004). Evidentemente, uma grande diversidade de especialistas é necessária para o estudo desse grupo, que pode ser abordado considerando sua função ecológica (Reynolds et al. 2002, Padisák et al. 2009), sua importância sanitária (Branco 1986, Cybis et al. 2006) e sua taxonomia stricto-sensu (Bicudo et al. 1996). A classificação destes organismos, assim como dos demais organismos vivos, está sendo revista e remodelada com o uso de técnicas de biologia molecular, alterando a história natural e filogenia até então conhecida (Reviers 2006, Cavalier-Smith 2009).

O número de espécies de organismos fitoplanctônicos é de aproximadamente 45.000 espécies (Chretiennot-Dinet \& Ricard 1991, Reviers 2006) englobando as espécies marinhas e de água doce, e com estimativas variando entre 72.500 espécies e um milhão de espécies (Guiry 2012). Segundo Agostinho et al. (2005), no Brasil há uma estimativa de 10.000 espécies de algas de água doce (incluindo as planctônicas e não planctônicas). Assim como outros grupos biológicos, o fitoplâncton tem seus estudos concentrados na região sul e sudeste do Brasil (Rocha 2006, Nabout et al. 2015). E, uma vez que o território brasileiro é pobre em ambientes límnicos naturais, os conhecimentos sobre o grupo estão concentrados em estudos limnológicos de reservatórios que tiveram seu maior desenvolvimento a partir da década de 80 (Barbosa et al. 1995).

No Mato Grosso do Sul os primeiros registros de organismos fitoplanctônicos foram feitos no início do século XX, ainda como estado de Mato Grosso, na cidade de Corumbá (Borges 1903 apud Martins 1980). Considerando o período após 1977, quando foi criado o estado de Mato Grosso do Sul, estudos sobre a comunidade fitoplanctônica concentraram-se no Pantanal na década de 1990 e início de 2000 (Espíndola et al. 1996, Oliveira \& Calheiros 2000), e na bacia do Paraná no século XXI, com estudos em represa urbana (Ferro et al. 2008, Lopes, 2009 e Silva 2012) e lagoa rural (Tremarin et al. 2013, 2014).

O fitoplâncton é essencial aos ecossistemas aquáticos, pois são produtores de biomassa e atuam na ciclagem de nutrientes, assim como na produção de oxigênio (Kasting $\&$ Siefert 2002). Devido a essa importância central em ecologia, os aspectos das populações e comunidades são a base para a compreensão dos ecossistemas aquáticos naturais e artificiais (Margalef 1983). Nesse sentido, a falta de especialistas em taxonomia exige a utilização de inferências indiretas, como a determinação da concentração de clorofila do ambiente. Estas medidas indiretas das populações fitoplanctônicas são relevantes, mas muito restritas para uma compreensão dos sistemas, impossibilitando a melhoria das modelagens ecológicas, de 
distribuição geográfica e da determinação das composições populacionais. Segundo Bicudo et al. (1996) estes problemas envolvendo identificação de organismos fitoplanctônicos estão na falta de especialistas (taxonomistas), assim como na falta de cursos de formação destes.

Esta revisão tem o objetivo de listar as espécies de organismos fitoplanctônicos registradas no estado de Mato Grosso do Sul, visando contribuir com futuros estudos taxonômicos, ecológicos e trabalhos aplicados à área de saneamento.

\section{MATERIAL E MÉTODOS}

A síntese dos dados sobre a ocorrência de espécies fitoplanctônicas no estado de Mato Grosso do Sul foi elaborada com base na revisão de trabalhos publicados sobre essa comunidade, como revistas científicas, resumos de congressos e dissertações de mestrado, até 2014 desconsiderando relatórios técnicos. Os nomes das espécies foram padronizados, com alterações nas citações antigas, as quais apresentavam identificação desatualizada, assim como acrescentados autores em citações específicas que não as continham. A nomenclatura das classes taxonômicas foi baseada em Reviers (2006). A distribuição geográfica dos pontos foi realizada tendo como base um mapa simplificado da hidrografia do estado elaborado a partir da carta cartográfica multirreferencial de Mato Grosso do Sul (1990).

\section{RESULTADOS E DISCUSSÃO}

O quadro 1 lista as espécies pertencentes às classes das populações de fitoplâncton registradas no estado do Mato Grosso do Sul agrupados por bacias, Paraguai e Paraná. Nesta listagem foram registradas dez classes: Bacillariophyceae, Cyanophyceae, Chlorophyceae, Chrysophyceae, Cryptophyceae, Dinophyceae, Euglenophyceae, Raphydophyceae, Zygnemaphyceae e Xanthophyceae. Dentre estas, as classes mais representativas foram: Bacillariophyceae, Cyanophyceae, Chlorophyceae, Euglenophyceae e Zygnemaphyceae.

A riqueza total no estado de Mato Grosso do Sul foi de 339 organismos identificados em nível de espécie e 190 identificados em nível de gênero, totalizando 529 espécimes. Na bacia do Paraná foram registrados 142 organismos em nível de espécie $(42 \%)$ e na bacia do Alto Paraguai foram registradas 197 (58\%), e 55 espécies foram comuns entre as duas bacias (16\%). A riqueza total do estado representa menos que $1 \%$ da diversidade de 10.000 algas de água doce registradas no Brasil (Agostinho et al. 2005). Este número foi altamente influenciado pelos estudos de Silva et al. (2000) no rio Paraguai com 197 espéciemes (37\%), estudo este publicado em resumo de encontro regional. Em periódicos científicos, Oliveira \& Calheiros (2000) publicaram a lista mais completa dos organismos fitoplanctônicos para o rio Paraguai, com 196 espécimes (37\%) e Train $\&$ Rodrigues (1998) publicaram lista semelhante para o rio Paraná, com coincidentes 196 espécimes (37\%).

As classes com as maiores riquezas identificadas em nível de espécies foram respectivamente: Chlorophyceae (66 espécies), Euglenophyceae (45 espécies), Cyanophyceae (34 espécies), Bacillariophyceae (25 espécies) e Zygnemaphyceae (16 espécies). As classes Cryptophyceae, Dinophyceae, Glaucophyceae e Raphydophyceae foram citados em trabalhos, mas não tiveram nenhum organismo identificado até espécie. Segundo Kruk et al. 2012 as classes Bacillariophyceae, Chlorophyceae, Cyanophyceae, Euglenophyceae, e Zygnemaphyceae estão comumente entre as classes mais representativas nos corpos de água doce.

A classe de maior representatividade nos corpos de água de Mato Grosso do Sul em número de espécies foi Chlorophyceae, que é estimada em 3500 espécies no Brasil (Rocha 2006) e em torno de 16000 no mundo, incluindo organismos marinhos e as macroalgas que é, também, a classe de maior representatividade em corpos de águas tropicais (Reviers 2006).

A segunda classe em número de espécies foi Euglenophyceae que é também complexa em termos de classificação (Reviers 2006). Bicudo \& Menezes (2010) levantaram 311 espécies de Euglenophyeae para o Brasil e Reviers (2006) contabilizou 1050 táxons no mundo. No estado de Mato Grosso do Sul o maior número de espécie foi registrado na bacia do Paraná, devido ao maior esforço amostral efetuado nesse ambiente. Esta classe tem enorme potencial de ser acrescida em um grande número de táxons no estado de Mato Grosso do Sul, se considerarmos a grande extensão e diversidade de áreas inundáveis no Pantanal que é centenas de vezes maior e muito mais complexa que a planície do Paraná (Tundisi \& Matsumura-Tundisi 2008).

As outras classes com grande riqueza foram na sequência, Cyanophyceae, Bacillariophyceae e Zygnemaphyceae. A classe Cyanophyceae está representada por aproximadamente 500 espécies no estado de São Paulo (Rocha 2006), enquanto que no Mato Grosso do Sul foram registradas 34 espécies, $7 \%$ do número de espécies do estado de São Paulo. O Estudo realizado por Santos \& Sant'Anna (2010) é o único com Cyanophyceae na região do pantanal da Nhecolândia no Mato Grosso do Sul. A classe Bacillariophyceae teve a listagem feita para o estado do Paraná analisando mais de 50 anos de registros distribuídos em 88 trabalhos, contabilizando 1004 táxons (Tremarin et al. 2009). Silva et al. (2011) registrou 503 táxons para a região centro-oste, incluindo 23 espécies no estado do Mato Grosso do Sul, e com os dois trabalhos específicos da classe realizados por Temarin et al. $(2013,2014)$ este número foi acrescido de duas novas espécies, totalizando 25 espécies para o estado. A classe Zygnemaphyceae, possui 2700 espécies catalogadas no mundo (Reviers 2006). E no Brasil não há levantamentos ou inventários completos para esta classe (Rocha 2006). Dentro desta, a família Desmidiacea foi inventariada no estado de São Paulo com um total de 450 espécies (Bicudo et al. 1996), portanto a lista de espécies de Mato Grosso do Sul é relativamente pequena com 16 espécies para a classe. 
Quadro 1. Espécies de fitoplâncton registradas no estado de Mato Grosso do Sul nas duas grandes bacias hidrográficas, Paraguai (1-7) e Paraná (8-12) com suas respectivas referências: 1 (Martins 1980); 2 (Oliveira \& Calheiros 2000); 3 (Silva et al. 2002 ); 4 (Domitrovic 2002); 5 (Malone et al. 2008); 6 (Vieira et al. 2009); 7 (Santos \& Sant'Anna 2010); 8 (Train \& Rodrigues 1998); 9 (Ferro et al. 2008 ); 10 (Lopes 2009 ); 11 (Silva 2012); 12 (Tremarin et al. 2013); 13 (Tremarin et al. 2014). Os números entre parênteses após o nome do gênero são número de espécies.

\begin{tabular}{|c|c|c|c|c|c|c|c|c|c|c|c|c|c|}
\hline \multirow{2}{*}{$\begin{array}{l}\text { Bacias } \\
\text { Táxons }\end{array}$} & \multicolumn{7}{|c|}{ Paraguai } & \multicolumn{6}{|c|}{ Paraná } \\
\hline & 1 & 2 & 3 & 4 & 5 & 6 & 7 & 8 & 9 & 10 & 11 & 12 & 13 \\
\hline \multicolumn{14}{|l|}{ Cyanophyceae } \\
\hline \multicolumn{14}{|l|}{ Anabaena cf. ambigua Rao. } \\
\hline A. circinalis $\mathrm{Rab}$. & & $\mathrm{X}$ & $\mathrm{X}$ & & & & & $\mathrm{X}$ & & & & & \\
\hline A. planctonica Brunnth. & & & & $\mathrm{X}$ & & & & & & & & & \\
\hline A. solitaria Kom. & & & & & & & & $\mathrm{X}$ & & & & & \\
\hline A. spiroides Kleb. & & & & $\mathrm{X}$ & & & & $\mathrm{X}$ & & & & & \\
\hline Anabaena spp . (2) Saint-Vincent ex Born. \& Flah. & & $\mathrm{X}$ & $\mathrm{X}$ & & & & & & & & & & \\
\hline Anabaenopsis cunningtonii Taylor & & & & & & & $\mathrm{X}$ & & & & & & \\
\hline A. elenkinii Miller. & & & & & & & $X$ & & & & & & \\
\hline \multicolumn{14}{|l|}{ Aphanizomenon gracile Lemm. } \\
\hline Aphanizomenon spp. (2) Morr. ex Born. \& C.Flah. & & & & & & & $X$ & & & $\mathrm{X}$ & $\mathrm{X}$ & & \\
\hline A. elachista W. West \& G. S. West & & & & $\mathrm{X}$ & & & & & & & & & \\
\hline Aphanocapsa sp. Nag. & & & & & & $\mathrm{X}$ & & & & $\mathrm{X}$ & & & \\
\hline Aphanothece sp. Nag. & & & & & & $\mathrm{X}$ & & & & & & & \\
\hline Arthrospira platensis (Nordst.) Gom. & & & & & $\mathrm{X}$ & & $\mathrm{X}$ & & & & & & \\
\hline Calothrix cf. flahaultii Frémy & & & & & & & $X$ & & & & & & \\
\hline Chroococcales (1) & & & & & & & & & & $\mathrm{X}$ & & & \\
\hline Chroococcus sp. Nag. & & $\mathrm{X}$ & & & & & & & & & & & \\
\hline Coelomoron tropicale Senn. \& Kom. & & & & & & & $\mathrm{X}$ & & & & & & \\
\hline Coelosphaerium confertum W. \& G.S.West & & $\mathrm{X}$ & & & & & & & & & & & \\
\hline Coelosphaerium spp. (2) Nag. & & & & & & $\mathrm{X}$ & & & & & & & \\
\hline Cylindrospermopsis (Wolosz.) Seen. \& Sub. & & & & & & & & & & $\mathrm{X}$ & & & \\
\hline C. raciborskii (Wolosz.) Seen. \& Sub. & & & & $\mathrm{X}$ & & & & $\mathrm{X}$ & & & & & \\
\hline Geitlerinema sp. (Anag \& Kom.) Anag. & & & & & & $\mathrm{X}$ & & & & & & & \\
\hline G. amphybium (Gom.) & & & & & & & & & $X$ & $\mathrm{X}$ & & & \\
\hline Glaucospira sp. Lagerh. & & & & & & & $X$ & $\mathrm{X}$ & & & & & \\
\hline Gloeocapsa sp. Kutz. & & $\mathrm{X}$ & & & & & & & & & & & \\
\hline J. subtilissimum (De Toni) Anag. \& Kom. & & & & & & & $\mathrm{X}$ & & & & & & \\
\hline Johannesbaptistia sp. De Toni & & & & & & & & & & $\mathrm{X}$ & & & \\
\hline Leptolyngbya cf. hypolimnetica (Camp.) Anag. & & & & & $\mathrm{X}$ & & & & & & & & \\
\hline L. martensiana Menegh. & & & & & & & & $\mathrm{X}$ & & & & & \\
\hline L. limnetica Lemm. & & & & & & & & $\mathrm{X}$ & & & & & \\
\hline Lyngbya sp. Agard. ex Gom. & & & & & & & & & & $\mathrm{X}$ & & & \\
\hline Merismopaedia tenuissima Lemm. & & & & & & & & & & $\mathrm{X}$ & & & \\
\hline M. glauca (Ehr.) Kütz. & & & & & & & & $\mathrm{X}$ & & & & & \\
\hline M. punctata Mey. & & & $\mathrm{X}$ & & & & & & & & & & \\
\hline M. punctata Mey. & & $\mathrm{X}$ & & & & & & & & & & & \\
\hline M. tenuissima Lemm. & & & & $\mathrm{X}$ & & & & $\mathrm{X}$ & & & & & \\
\hline Merismopedia spp. (2) Mey. & & $\mathrm{X}$ & $\mathrm{X}$ & & & $\mathrm{X}$ & & & & & & & \\
\hline Microcystis aeruginosa Kützing & & $\mathrm{X}$ & $\mathrm{X}$ & & & & & $\mathrm{X}$ & & & & & \\
\hline Myxobaktron sp. Schmid. & & & & & & & $\mathrm{X}$ & & & & & & \\
\hline Nostoc sp. Vauch. ex Bor. \& Flah. & & & $\mathrm{X}$ & & & & & & & & & & \\
\hline Ocilatoriales (1) & & & & & & & & & & $\mathrm{X}$ & & & \\
\hline Oscillatoria sancta (Kutz.) Gom. & & & & & & & & $\mathrm{X}$ & & & & & \\
\hline Oscillatoria spp. (4) & & $\mathrm{X}$ & $\mathrm{X}$ & & & $\mathrm{X}$ & & & & & $\mathrm{X}$ & & \\
\hline Phormidium ambiguum Gom. ex Gom. & & & & & $\mathrm{X}$ & & $X$ & & & & & & \\
\hline Phormidium sp. Kutz. ex Gom. & & & & & & $\mathrm{X}$ & $\mathrm{X}$ & & & & & & \\
\hline P. tenue (Ag. ex Gom) Anag. \& Kom. & & & & & & & & & & & $\mathrm{X}$ & & \\
\hline P. tergestinum (Gom.) Anag. \& Kom. & & & & & & & $\mathrm{X}$ & & & & & & \\
\hline Planktolyngbya sp. Anag. \& Kom. & & & & & & & & & $\mathrm{X}$ & $\mathrm{X}$ & & & \\
\hline Planktolyngbya contorta (Lemm.) Anagn. \& Kom. & & & & $\mathrm{X}$ & & & & & & & & & \\
\hline P. limnetica (Lemm.) Kom.-Legn. \& Cronb. & & & & & $\mathrm{X}$ & & $X$ & & & & & & \\
\hline P. subtilis (W. West) Anagn. \& Kom. & & & & $\mathrm{X}$ & & & & & & & & & \\
\hline Planktothrix agardhii (Gom.) Anag. \& Kom. & & & & & & & $\mathrm{X}$ & & & $\mathrm{X}$ & & & \\
\hline
\end{tabular}


Quadro 1. Cont.

\begin{tabular}{|c|c|c|c|c|c|c|c|c|c|c|c|c|c|}
\hline \multirow{2}{*}{$\begin{array}{l}\text { Bacias } \\
\text { Táxons }\end{array}$} & \multicolumn{7}{|c|}{ Paraguai } & \multicolumn{6}{|c|}{ Paraná } \\
\hline & 1 & 2 & 3 & 4 & 5 & 6 & 7 & 8 & 9 & 10 & 11 & 12 & 13 \\
\hline P. isothrix (Skuja) Kom \& Kom & & & & & & & $\mathrm{X}$ & & & & $\mathrm{X}$ & & \\
\hline Planktothrix sp. Anag. \& Kom. & & & & & & $\mathrm{X}$ & & & & & & & \\
\hline Pseudanabaena limnetica (Lemm.) Kom. & & & & & & & $\mathrm{X}$ & & & & & & \\
\hline Pseudanabaenasubg. Ilyonema Anag. \& Kom. & & & & & $\mathrm{X}$ & & & & & & & & \\
\hline Pseudanabaena spp. (2) Lauterb. & & & & & $\mathrm{X}$ & & & & & $\mathrm{X}$ & & & \\
\hline Radiocystis sp. Sku. & & & & & & & & & & $\mathrm{X}$ & & & \\
\hline Raphidiopsis mediterranea Sku. & & & & & & & & $\mathrm{X}$ & & & & & \\
\hline Romeria caruaru Komárek et al. & & & & & & & $\mathrm{X}$ & & & & & & \\
\hline R. gracilis (Koczw.) Koczw. ex. Geit. & & & & & & & & & & $\mathrm{X}$ & & & \\
\hline R. leopolinenses (Racib.) Koczw. & & & & & & & & & & $\mathrm{X}$ & & & \\
\hline R. victoriae Komárek \& Cronberg & & & & & & & $\mathrm{X}$ & & & & & & \\
\hline Snowella sp. Elenk. & & & & & & & & & & $\mathrm{X}$ & & & \\
\hline Sphaerocavum sp. Azev. \& Sant. & & & & & & $\mathrm{X}$ & & & & & & & \\
\hline Spirulina subsalsa Gomont, Ann. & & & & & & & $\mathrm{X}$ & & & & & & \\
\hline Spirulina subtilissima Gomont, Ann. & & & & & & & $\mathrm{X}$ & & & & & & \\
\hline S. cf. nidulans Kom. in Bourrely & & & & & & & $\mathrm{X}$ & & & & & & \\
\hline S. sigmoideus (Moore \& Carter) Kom. & & & & & & & $\mathrm{X}$ & & & & & & \\
\hline Synechocystis aquatilis Sauv., Bull. & & & & & & & $\mathrm{X}$ & & & & & & \\
\hline Synechocystis sp. Sauv. & & & & & & & & & & $\mathrm{X}$ & & & \\
\hline Chloroplyceae & & & & & & & & & & & & & \\
\hline Actinastrum aciculare Playf. & & $\mathrm{X}$ & & & & & & & & & & & \\
\hline A. gracillimum G. M. Smith & & & & & & & & $\mathrm{X}$ & & & & & \\
\hline A. hantzschii Lag. & & & $\mathrm{X}$ & & & & & & & $\mathrm{X}$ & & & \\
\hline A. hantzschii Lag. & & $\mathrm{X}$ & & & & & & & & & & & \\
\hline Actinastrum spp. (2) Lagerh. & & & $\mathrm{X}$ & & & & & & & & & & \\
\hline A. cf fusiformis Corda & & & & & & & & & & $\mathrm{X}$ & & & \\
\hline Ankistrodesmus sp. Cor. & & & & & & $\mathrm{X}$ & & & & & & & \\
\hline A. fusiformes Cor. & & & & & & & & $\mathrm{X}$ & & & & & \\
\hline A. gracilis (Rein.) Korsik. & & $\mathrm{X}$ & $\mathrm{X}$ & & & & & & & $\mathrm{X}$ & & & \\
\hline A. judayi (G. M. Smith) Fott & & & $\mathrm{X}$ & & & & & & & & & & \\
\hline Botryococcus sp. Kutz. & & & & & & $\mathrm{X}$ & & & & & & & \\
\hline B. protuberans West \& G. S. West. & & & & & & & & $\mathrm{X}$ & & & & & \\
\hline Chlamydomonas microsphaera Pasch. \& Jah. & & & $\mathrm{X}$ & & & & & & & & & & \\
\hline Chlamydomonas spp. (2) Ehr. & & & & & & & & $\mathrm{X}$ & & & & & \\
\hline Chlorella ellipsoidea Gern. & & & $\mathrm{X}$ & & & & & & & & & & \\
\hline Chlorococcales (1) & & & & & & & & & & $\mathrm{X}$ & & & \\
\hline Chlorococcum sp. Menegh. & & & & & & & & & & $\mathrm{X}$ & & & \\
\hline Chloromonas acidophila (Nyg.) Gerl. \& Ettl & & & $\mathrm{X}$ & & & & & & & & & & \\
\hline C. gracilis (Matwienko) Ettl & & & $\mathrm{X}$ & & & & & & & & & & \\
\hline Choricystis cylindracea Hind. & & & $\mathrm{X}$ & & & & & & & & & & \\
\hline C. minor (Skuja) Fott & & & $\mathrm{X}$ & & & & & & & & & & \\
\hline Closteriopsis acicularis (G. M. Sm.) Belc \& Sw. & & & & & & & & $\mathrm{X}$ & & & & & \\
\hline Closteriopsis spp. (3) Lemm. & & $\mathrm{X}$ & & & & $\mathrm{X}$ & & & & $\mathrm{X}$ & & & \\
\hline Coccomonas platyformis Jane & & & $\mathrm{X}$ & & & & & & & & & & \\
\hline Coelastrum cf indicum Turn. & & & & & & & & & & $\mathrm{X}$ & & & \\
\hline C. microporum (Nag.) Bohl. & & $\mathrm{X}$ & $\mathrm{X}$ & & & & & & & $\mathrm{X}$ & & & \\
\hline C. microporum Näg. & & & $\mathrm{X}$ & & & & & & & & & & \\
\hline Coelastrum sp. Nag. & & & & & & $\mathrm{X}$ & & & & & & & \\
\hline C. reticulatum (Dang.) Senn. & & & $\mathrm{X}$ & & & & & & & & & & \\
\hline C. planconvexa Hind. & & & & & & & & & & $\mathrm{X}$ & & & \\
\hline Coenochloris spp. (3) Kors. & & & $\mathrm{X}$ & & & & & & & $\mathrm{X}$ & & & \\
\hline Coenocystis sp. (2) Kors. & & & & & & & & & & $\mathrm{X}$ & & & \\
\hline C. mucronata (G. M. Smith) Kom. & & & $\mathrm{X}$ & & & & & & & & & & \\
\hline C. quadrata Morr. & & & $\mathrm{X}$ & & & & & & & & & & \\
\hline C. rectangularis (Näg.) Kom. & & & $\mathrm{X}$ & & & & & $\mathrm{X}$ & & & & & \\
\hline Crucigenia spp. (2) Morr. & & & & & & & & $\mathrm{X}$ & & $\mathrm{X}$ & & & \\
\hline
\end{tabular}


Quadro 1. Cont.

\begin{tabular}{|c|c|c|c|c|c|c|c|c|c|c|c|c|c|}
\hline \multirow{2}{*}{$\begin{array}{l}\text { Bacias } \\
\text { Táxons }\end{array}$} & \multicolumn{7}{|c|}{ Paraguai } & \multicolumn{6}{|c|}{ Paraná } \\
\hline & 1 & 2 & 3 & 4 & 5 & 6 & 7 & 8 & 9 & 10 & 11 & 12 & 13 \\
\hline C. tetrapedia (Kirch.) W. \& G.S. West & & $\mathrm{X}$ & $\mathrm{X}$ & & & & & $\mathrm{X}$ & & & & & \\
\hline C. pulchra (Nageli) Bohlin & & $\mathrm{X}$ & & & & & & & & & & & \\
\hline Crucigeniella spp. (2) Lemm. & & & $\mathrm{X}$ & & & & & & & $\mathrm{X}$ & & & \\
\hline Deflandre Oocystis lacustris Chodat & & $\mathrm{X}$ & & & & & & & & & & & \\
\hline D. cf. communis E.Hegew. & & & & & & & & & & $\mathrm{X}$ & & & \\
\hline D. cf. spinosus (Chod.) E.Hegew. & & & & & & & & & & $\mathrm{X}$ & & & \\
\hline D. intermedius (Chod.) Hegew. & & & & & & & & & & $\mathrm{X}$ & & & \\
\hline D. intermedius var. acutispinus (Roll) Hegew. & & & & & & & & & & $\mathrm{X}$ & & & \\
\hline Dictyosphaerium sp. Nag. & & & $\mathrm{X}$ & & & & & & & & & & \\
\hline Dictyosphaerium pulchellum Wood & & $\mathrm{X}$ & & & & & & $\mathrm{X}$ & & $\mathrm{X}$ & & & \\
\hline D. ehrenbergianum Nag. & & $\mathrm{X}$ & $\mathrm{X}$ & & & & & $\mathrm{X}$ & & & & & \\
\hline Dictyosphaerium sp. Nag. & & & & & & $\mathrm{X}$ & & & & & & & \\
\hline D. tetrachotomun Printz & & & $\mathrm{X}$ & & & & & & & & & & \\
\hline Didymocystis bicellularis (Chod.) Kom. & & & $\mathrm{X}$ & & & & & & & & & & \\
\hline Didymocystis sp. Kors. & & & & & & $\mathrm{X}$ & & & & & & & \\
\hline Elakatothrix sp. Will. & & & & & & & & $\mathrm{X}$ & & & & & \\
\hline Eudorina elegans Ehr. & & $\mathrm{X}$ & $\mathrm{X}$ & & & & & $\mathrm{X}$ & & & & & \\
\hline Eudorina spp. (2) Ehr. & & & $\mathrm{X}$ & & & & & & & & & & \\
\hline Eutetramorus fottii (Hind.'ak) Kom. & & $\mathrm{X}$ & $\mathrm{X}$ & & & & & & & $\mathrm{X}$ & & & \\
\hline E. planctonicus (Kors.) Bourr. & & & & & & & & $\mathrm{X}$ & & & & & \\
\hline E. tetrasporus Kom. & & & & & & & & & & $\mathrm{X}$ & & & \\
\hline Gloecapsa sp. Kutz. & & & $\mathrm{X}$ & & & & & & & & & & \\
\hline G. radiata Chod. & & $\mathrm{X}$ & $\mathrm{X}$ & & & & & & & $\mathrm{X}$ & & & \\
\hline Gonium pectorale Mull. & & & $\mathrm{X}$ & & & & & & & & & & \\
\hline Gonium sp. Mull. & & & $\mathrm{X}$ & & & & & & & & & & \\
\hline Keratococcus bicaudatus (A. Braun) Peter. & & $\mathrm{X}$ & & & & & & & & & & & \\
\hline Kirchneriella spp. (2) Schm. & & & $\mathrm{X}$ & & & & & & & $\mathrm{X}$ & & & \\
\hline K. lunaris (Kirchn.) Moeb. & & & $\mathrm{X}$ & & & & & & & & & & \\
\hline K. obesa (W. W.) Schm. & & & & & & & & $\mathrm{X}$ & & & & & \\
\hline K. roselata Hind. & & & & & & & & $\mathrm{X}$ & & & & & \\
\hline Lagerheimia chodati Bern. & & $\mathrm{X}$ & & & & & & & & & & & \\
\hline Micractinium bornhemiense (Conr.) Kors. & & & $\mathrm{X}$ & & & & & & & $\mathrm{X}$ & & & \\
\hline M. pusillum Fres. & & $\mathrm{X}$ & $\mathrm{X}$ & & & & & & & $\mathrm{X}$ & & & \\
\hline Micractinium sp. Fres. & & & $\mathrm{X}$ & & & & & & & & & & \\
\hline Monoraphidium arcuatum (Kors) Hind. & & $\mathrm{X}$ & & & & & & $\mathrm{X}$ & & $\mathrm{X}$ & & & \\
\hline M. contortum (Thur.) Kom. - Legn. & & & & & & & & $\mathrm{X}$ & & $\mathrm{X}$ & & & \\
\hline M. convolutum (Cor.) Kom.-Legn. & & & $\mathrm{X}$ & & & & & $\mathrm{X}$ & & $\mathrm{X}$ & & & \\
\hline M. fontinali Hind. & & & & & & & & $\mathrm{X}$ & & & & & \\
\hline M. griffithii (Berk.) Kom.-Legn. & & $\mathrm{X}$ & & & & & & & & $\mathrm{X}$ & & & \\
\hline M. irregulare (G. M. Sm.) Kom.-Legn. & & & $\mathrm{X}$ & & & & & $\mathrm{X}$ & & & & & \\
\hline Monoraphidium irregulare (G.M.Smit.) Kom.-Legn. & & & & & & & & & & $\mathrm{X}$ & & & \\
\hline M. komarkovae Nyg. & & & & & & & & $\mathrm{X}$ & & & & & \\
\hline M. minutum (Näg.) Kom.-Legn. & & & $\mathrm{X}$ & & & & & $\mathrm{X}$ & & $\mathrm{X}$ & & & \\
\hline Monoraphidium spp. (3) Kom.-Legn. & & & $\mathrm{X}$ & & & $\mathrm{X}$ & & & & $\mathrm{X}$ & & & \\
\hline M. tortile (W.\&G.S. West) Kom.- Legn. & & $\mathrm{X}$ & $\mathrm{X}$ & & & & & $\mathrm{X}$ & & $\mathrm{X}$ & & & \\
\hline Nephrochlamys spp. (2) Kor. & & & & & & $\mathrm{X}$ & & & & & & & \\
\hline N. lunatum $\mathrm{W}$. West & & & & & & & & $\mathrm{X}$ & & & & & \\
\hline O. lacustris Chod. & & & $\mathrm{X}$ & & & & & $\mathrm{X}$ & & & & & \\
\hline O. marssonii Lemm. & & & $\mathrm{X}$ & & & & & & & & & & \\
\hline Oocystis sp. Nag. \& Bra. & & & & & & & & & & $\mathrm{X}$ & & & \\
\hline Pachycladella sp. Silv. & & & & & & & & & & $\mathrm{X}$ & & & \\
\hline Pachycladella umbrina (G.M.Sm.) Silv. & & & $\mathrm{X}$ & & & & & & & & & & \\
\hline Paradoxia multiseta Svir. & & & $\mathrm{X}$ & & & & & & & & & & \\
\hline P. duplex Mey. var. duplex & & & $\mathrm{X}$ & & & & & & & $\mathrm{X}$ & & & \\
\hline P. duplex var. gracillimum W. West. \& G. S. West & & & $\mathrm{X}$ & & & & & & & & & & \\
\hline P. simplex var. simplex Mey. & & & $\mathrm{X}$ & & & & & & & $\mathrm{X}$ & & & \\
\hline
\end{tabular}


Quadro 1. Cont.

\begin{tabular}{|c|c|c|c|c|c|c|c|c|c|c|c|c|c|}
\hline \multirow{2}{*}{$\begin{array}{l}\text { Bacias } \\
\text { Táxons }\end{array}$} & \multicolumn{7}{|c|}{ Paraguai } & \multicolumn{6}{|c|}{ Paraná } \\
\hline & 1 & 2 & 3 & 4 & 5 & 6 & 7 & 8 & 9 & 10 & 11 & 12 & 13 \\
\hline P. tetras (Ehr.) Ralfs & & $\mathrm{X}$ & $\mathrm{X}$ & & & & & $\mathrm{X}$ & & & & & \\
\hline Pteromonas rectangularis Lemm. & & & $\mathrm{X}$ & & & & & & & & & & \\
\hline Q. closterioides (Bohl.) Printz & & & & & & & & $\mathrm{X}$ & & & & & \\
\hline S. acuminatus (Lagrh.) Chod. & & $\mathrm{X}$ & $\mathrm{X}$ & & & & & & & $\mathrm{X}$ & & & \\
\hline S. acutus Mey. & & & & & & & & $\mathrm{X}$ & & & & & \\
\hline S. bicaudatus Dedus. & & & $\mathrm{X}$ & & & & & $\mathrm{X}$ & & & & & \\
\hline S. brasiliensis Bohl. & & & $\mathrm{X}$ & & & & & & & & & & \\
\hline S. cf arthrodesmiformis Schröder & & & & & & & & & & $\mathrm{X}$ & & & \\
\hline S.cf disciformis (Chod.) Fott \& Kom. & & & & & & & & & & $\mathrm{X}$ & & & \\
\hline S. denticulatus Lagerheim & & & $\mathrm{X}$ & & & & & & & & & & \\
\hline Scenedesmus ecornis (Ehr.) Chod. & & & $\mathrm{X}$ & & & & & & & $\mathrm{X}$ & & & \\
\hline S. ellipticus Cor. & & & & & & & & $\mathrm{X}$ & & & & & \\
\hline S. quadricauda (Turp.) Bréb. sensu Chod. & & & $\mathrm{X}$ & & & & & $\mathrm{X}$ & & & & & \\
\hline Scenedesmus spp. (4) Mey. & & $\mathrm{X}$ & & & & $\mathrm{X}$ & & & & & & & \\
\hline Schroederia antillarum Kom. & & & $\mathrm{X}$ & & & & & & & $\mathrm{X}$ & & & \\
\hline S. nitzschioides (G. S. West) Kors. & & & & & & & & & & & & & \\
\hline S. setigera (Schröd.) Lemm. & & & & & & & & $\mathrm{X}$ & & $\mathrm{X}$ & & & \\
\hline Schroederiella africana Wolosz. & & & $\mathrm{X}$ & & & & & & & & & & \\
\hline S. gracile Reins. & & & & & & & & $\mathrm{X}$ & & & & & \\
\hline Selenastrum sp. Reins. & & & $\mathrm{X}$ & & & & & & & & & & \\
\hline Selenodyctium brasiliense Uherk. \& Schm. ex. Com. \& Kom. & & & $\mathrm{X}$ & & & & & & & & & & \\
\hline S. planctonica (Kors.) Bourr. & & & $\mathrm{X}$ & & & & & & & & & & \\
\hline S. schroeteri Chod. & & & $\mathrm{X}$ & & & & & & & & & & \\
\hline Synedra sp. Ehr. & & & $\mathrm{X}$ & & & & & & & & & & \\
\hline Teilingia wallichii var. borgei (Grönbl.) Först. & & & $\mathrm{X}$ & & & & & & & & & & \\
\hline Tetrachlorella alternans (G. M. Sm.) Kors. & & & $\mathrm{X}$ & & & & & & & & & & \\
\hline Tetraedron caudatum (Corda) Hansg. & & & $\mathrm{X}$ & & & & & & & & & & \\
\hline Tetraedron sp. Kutz. & & & & & & $\mathrm{X}$ & & & & & & & \\
\hline T. minimum (A. Br.) Hansg. & & & & & & & & $\mathrm{X}$ & & & & & \\
\hline T. heteracanthum (Nordst.) Chod. & & & $\mathrm{X}$ & & & & & & & & & & \\
\hline T. schmidlei (Schrod.) Fott \& Kovac. & & & $\mathrm{X}$ & & & & & & & & & & \\
\hline T. setigera (Arch.) G. M. Sm. & & & $\mathrm{X}$ & & & & & & & & & & \\
\hline Treubaria sp. Bern. & & & $\mathrm{X}$ & & & & & & & & & & \\
\hline Volvocales (2) & & & & & & & & & & $\mathrm{X}$ & & & \\
\hline Volvox spp. (3) L. & & & $\mathrm{X}$ & & & & & & & $\mathrm{X}$ & & & \\
\hline Euglenoplyceae & & & & & & & & & & & & & \\
\hline Euglena acus Ehr. & & $\mathrm{X}$ & $\mathrm{X}$ & $\mathrm{X}$ & & & & $\mathrm{X}$ & & & & & \\
\hline E. allorgei Defl. & & & & $\mathrm{X}$ & & & & & & & & & \\
\hline E. tripteris (Duj.) Klebs & & & & & & & & $\mathrm{X}$ & & & & & \\
\hline E. oxyuris $\mathrm{Schm}$. & & & $\mathrm{X}$ & $\mathrm{X}$ & & & & $\mathrm{X}$ & & & & & \\
\hline Euglena spp. (5) Ehr. & & $\mathrm{X}$ & & $\mathrm{X}$ & & $\mathrm{X}$ & & & & $\mathrm{X}$ & & & \\
\hline E. spirogyra Ehr. & & & $\mathrm{X}$ & & & & & & & & & & \\
\hline E. tripteris (Duj.) Klebs & & & & $\mathrm{X}$ & & & & & & & & & \\
\hline Lepocinclis acus (O.F.Mull.) Mar. \& Melk. & & & & & & & & & & $\mathrm{X}$ & & & \\
\hline L. fusiformis (Carter) Lemm. Emend. Conr. & & & & & & & & $\mathrm{X}$ & & & & & \\
\hline L. ovum (Ehr.) Lemm. & & & & $\mathrm{X}$ & & & & $\mathrm{X}$ & & $\mathrm{X}$ & & & \\
\hline L. oxyuris (Brons.) Schm. & & & & & & & & & & $\mathrm{X}$ & & & \\
\hline L. pseudonayalii Tell \& Zal. & & & & $\mathrm{X}$ & & & & & & & & & \\
\hline L. texta var. texta (Duj.) Lemm. Emend. Conr. & & & & & & & & $\mathrm{X}$ & & & & & \\
\hline Lepocinclis sp. Per. & & & $\mathrm{X}$ & & & & & & & & & & \\
\hline P. agilis Sku. & & & & $\mathrm{X}$ & & & & & & & & & \\
\hline P. cf. gigas Cunh. & & & & & & & & $\mathrm{X}$ & & & & & \\
\hline P. cf. onix Pochm. & & & & $\mathrm{X}$ & & & & & & & & & \\
\hline P. horridus Pochm. & & & & & & & & $\mathrm{X}$ & & & & & \\
\hline P. longicauda (Ehr.) Duj. var. tortus Lemm. & & & & & & & & & & $\mathrm{X}$ & & & \\
\hline P. longicauda var. attenuada (Pochm.) Hüb-Pest. & & & & & & & & & & & & & \\
\hline
\end{tabular}


Quadro 1. Cont.

\begin{tabular}{|c|c|c|c|c|c|c|c|c|c|c|c|c|c|}
\hline \multirow{2}{*}{$\begin{array}{l}\text { Bacias } \\
\text { Táxons }\end{array}$} & \multicolumn{7}{|c|}{ Paraguai } & \multicolumn{6}{|c|}{ Paraná } \\
\hline & 1 & 2 & 3 & 4 & 5 & 6 & 7 & 8 & 9 & 10 & 11 & 12 & 13 \\
\hline P. longicauda var. longicauda (Ehr.) Duj. & & & & & & & & $\mathrm{X}$ & & & & & \\
\hline P. longicauda var. torta Lemm. & & & & & & & & & & $\mathrm{X}$ & & & \\
\hline P. minutus (Playf.) Pochm. & & & & $X$ & & & & & & & & & \\
\hline P. orbicularis Hubn. & & $\mathrm{X}$ & & & & & & $\mathrm{X}$ & & $\mathrm{X}$ & & & \\
\hline P. pleuronectes (Muell.) Nitzs. ex Duj. & & & & & & & & $\mathrm{X}$ & & & & & \\
\hline P. pyrum (Ehr.) St. & & & & & & & & $\mathrm{X}$ & & & & & \\
\hline Phacus spp. (5) Duj. & & $\mathrm{X}$ & $\mathrm{X}$ & & & $\mathrm{X}$ & & & & $\mathrm{X}$ & & & \\
\hline P. suecicus Lemm. & & & & & & & & $\mathrm{X}$ & & & & & \\
\hline P. tortus (Lemm.) Skv. & & $\mathrm{X}$ & $\mathrm{X}$ & & & & & & & $\mathrm{X}$ & & & \\
\hline P. verrucoso (Lemm.) Defl. & & $\mathrm{X}$ & & & & & & & & & & & \\
\hline P. suecicus (Lemm.) Pascher \& Lemm. & & $\mathrm{X}$ & & & & & & & & & & & \\
\hline Strombomonas acuminata (Schm.) Defl. & & $\mathrm{X}$ & $\mathrm{X}$ & & & & & & & & & & \\
\hline S. ensifera (Dad.) Defl. & & & $\mathrm{X}$ & $\mathrm{X}$ & & & & & & & & & \\
\hline S. ensifera var. javanica Hüb-Pest & & & & & & & & & & & & & \\
\hline S. fluviatilis (Lemm.) & & $\mathrm{X}$ & $\mathrm{X}$ & & & & & & & & & & \\
\hline S. jaculata (Palmer) Defl. & & & & $\mathrm{X}$ & & & & & & & & & \\
\hline S. maxima (Skv.) Defl. & & & & $\mathrm{X}$ & & & & & & & & & \\
\hline S. ovalis (Playf.) Defl. & & & & $\mathrm{X}$ & & & & & & & & & \\
\hline Strombomonas spp. (3) Defl. & & $\mathrm{X}$ & $\mathrm{X}$ & & & & & & & $\mathrm{X}$ & & & \\
\hline S. treubii (Wol.) Defl. & & & & $\mathrm{X}$ & & & & & & & & & \\
\hline S. verrucosa var. zmiewika (Swir.) Defl. & & & & $\mathrm{X}$ & & & & & & & & & \\
\hline Trachelomonas abrupta var. minor Defl. & & & & $\mathrm{X}$ & & & & & & & & & \\
\hline T. acanthophora Stokes & & $\mathrm{X}$ & & $\mathrm{X}$ & & & & & & & & & \\
\hline T. armata Ehr. & & & $\mathrm{X}$ & & & & & & & $\mathrm{X}$ & & & \\
\hline T. armata var. steinii Lemm. Emend Defl. & & & & $\mathrm{X}$ & & & & & & & & & \\
\hline T. cervicula Stokes & & $\mathrm{X}$ & & $\mathrm{X}$ & & & & & & & & & \\
\hline T. curta da Cunha Emend Defl. var. curta & & & & $\mathrm{X}$ & & & & & & $\mathrm{X}$ & & & \\
\hline T. curta da Cunha Emend. Defl. var. minima Tell \& Zaloc. & & & & & & & & $\mathrm{X}$ & & & & & \\
\hline T. estriada Stokes & & $\mathrm{X}$ & & & & & & & & & & & \\
\hline T. gracillima Balech \& Dast. & & & & $\mathrm{X}$ & & & & & & & & & \\
\hline T. hispida (Perty) Stein Emend. Defl. var. coronata Lemm. & & & $\mathrm{X}$ & & & & & $\mathrm{X}$ & & $\mathrm{X}$ & & & \\
\hline T. hispida (Pet.) Stein Emend. Defl. & & $\mathrm{X}$ & & & & & & & & & & & \\
\hline T. multifacies Yacub. \& Brav. & & & & $\mathrm{X}$ & & & & & & & & & \\
\hline T. oblonga Lemm. & & $\mathrm{X}$ & & & & & & $\mathrm{X}$ & & & & & \\
\hline T. rugulosa Stein & & & & $\mathrm{X}$ & & & & $\mathrm{X}$ & & & & & \\
\hline T. sculpta Balech & & & & $\mathrm{X}$ & & & & & & & & & \\
\hline T. similis Stok. & & $\mathrm{X}$ & & & & & & & & & & & \\
\hline Trachelomonas spp. (6) Ehr. & & $\mathrm{X}$ & $\mathrm{X}$ & & & $\mathrm{X}$ & & & & & & & \\
\hline T. varians Defl. & & & & $\mathrm{X}$ & & & & & & & & & \\
\hline T. volvocina Ehr. & & $\mathrm{X}$ & $\mathrm{X}$ & & & & & & & & & & \\
\hline T. volvocina Ehr. var. volvocina & & & & $\mathrm{X}$ & & & & & & & & & \\
\hline T. volvocina var. derephora Conr. & & & & $\mathrm{X}$ & & & & & & & & & \\
\hline T. volvocinopsis & & & $\mathrm{X}$ & & & & & & & & & & \\
\hline T.. volvocinopsis Swir. & & $\mathrm{X}$ & & & & & & & & & & & \\
\hline T. bernardii Wol. & & $\mathrm{X}$ & & & & & & & & & & & \\
\hline T. cf. mucosa Svirenko & & $\mathrm{X}$ & & & & & & & & & & & \\
\hline Bacillariophyceae & & & & & & & & & & & & & \\
\hline Actinocyclus normanii (Greg. ex Grev.) Hust. & & & & $\mathrm{X}$ & & & & & & & & & \\
\hline A. ambigua (Grunow) Simonsen & & & & & & & & & & $\mathrm{X}$ & & $\mathrm{X}$ & \\
\hline A. distans (Ehr.) Simonsen & & $\mathrm{X}$ & & $\mathrm{X}$ & & & & & & & & & \\
\hline A. granulata (Ehr.) Sim. var. angustissima (O. Mull.) Sim. & & & & & & & & & & $\mathrm{X}$ & & & \\
\hline A. granulata (Ehr.) Simonsen & & & & $\mathrm{X}$ & & & & $\mathrm{X}$ & & $\mathrm{X}$ & & & \\
\hline A. granulata var. angustissima (O.F. Mull.) Simon. & & $\mathrm{X}$ & & & & & & & & & & & \\
\hline A. granulata var. granulata (Ehr.) Sim. & & $\mathrm{X}$ & & & & & & & & & & & \\
\hline A. herzogii (Lemm.) Sim. & & $\mathrm{X}$ & & $\mathrm{X}$ & & & & $\mathrm{X}$ & & & & & \\
\hline
\end{tabular}


Quadro 1. Cont.

\begin{tabular}{|c|c|c|c|c|c|c|c|c|c|c|c|c|c|}
\hline \multirow{2}{*}{$\begin{array}{l}\text { Bacias } \\
\text { Táxons }\end{array}$} & \multicolumn{7}{|c|}{ Paraguai } & \multicolumn{6}{|c|}{ Paraná } \\
\hline & 1 & 2 & 3 & 4 & 5 & 6 & 7 & 8 & 9 & 10 & 11 & 12 & 13 \\
\hline A. italica (Ehrenberg) Sim. & & & & $\mathrm{X}$ & & & & & & & & & \\
\hline A. veraluciae & & & & & & & & & & & & & $\mathrm{X}$ \\
\hline Aulacoseira sp. Thw. & & & & & & & & & & $\mathrm{X}$ & & & \\
\hline Cocconeis placentula var. acuta Meist. & & & & $\mathrm{X}$ & & & & & & & & & \\
\hline Cyclotella meneghiniana Kutz. & & $\mathrm{X}$ & & $\mathrm{X}$ & & & & & & & & & \\
\hline \multicolumn{14}{|l|}{ C. pseudostelligera Hust. } \\
\hline Cyclotella spp. (3) (Kutz.) Bréb. & & & & $\mathrm{X}$ & & & & & & $\mathrm{X}$ & & & \\
\hline C. stelligera $\mathrm{Cl}$ \& \& Grun. & & & & $\mathrm{X}$ & & & & $\mathrm{X}$ & & & & & \\
\hline Cymbella spp. (3) Agard. & & & & $\mathrm{X}$ & & & & & & & & & \\
\hline Eunotia bilunaris (Ehr.) Mills & & & & $\mathrm{X}$ & & & & & & & & & \\
\hline E. camelus Ehr. & & & & & & & & $\mathrm{X}$ & & & & & \\
\hline E. curvata (Kütz.) Lagerstedt & & $\mathrm{X}$ & & $\mathrm{X}$ & & & & & & & & & \\
\hline E. didyma var. gibbosa (Grun.) Hust. & & & & $\mathrm{X}$ & & & & & & & & & \\
\hline E. monodon Ehr. & & & & $\mathrm{X}$ & & & & $\mathrm{X}$ & & & & & \\
\hline Eunotia spp. (3) Ehr. & & $\mathrm{X}$ & & $\mathrm{X}$ & & & & & & & & & \\
\hline Fragilaria capucina Desm. & & & & & & & & $\mathrm{X}$ & & & & & \\
\hline F. crotonensis Kitton & & & & & & & & & & $\mathrm{X}$ & & & \\
\hline Frustulia spp. (2) Rab. & & & & $\mathrm{X}$ & & & & & & & & & \\
\hline G. augur var. turris (Ehr.) Lange-Bert. & & & & $\mathrm{X}$ & & & & & & & & & \\
\hline Gyrosigma acuminatum (Kütz.) Rab. & & & & $\mathrm{X}$ & & & & & & & & & \\
\hline N. cuspidata (Kütz.) Kütz. & & & & $\mathrm{X}$ & & & & & & & & & \\
\hline Navicula spp. (5) Saint-Vinc. & & $\mathrm{X}$ & & $\mathrm{X}$ & & & & & & & & & \\
\hline Nitzschia acicularis Sm. & & & & $\mathrm{X}$ & & & & $\mathrm{X}$ & & & & & \\
\hline N. gracilis Hantz. ex Rabenh. & & & & & & & & $\mathrm{X}$ & & & & & \\
\hline N. levidensis var. victoriae (Grun.) Chol. & & & & $\mathrm{X}$ & & & & & & & & & \\
\hline N. palea (Kütz.) W. Sm. & & & & & & & & $\mathrm{X}$ & & & & & \\
\hline Nitzschia spp. (3) Saint-Vinc. & & $\mathrm{X}$ & & $\mathrm{X}$ & & & & $\mathrm{X}$ & & & & & \\
\hline Pennales spp. (6) & & & & & & & & & & $\mathrm{X}$ & & & \\
\hline Pinnularia acrosphaeria $\mathrm{Sm}$. & & & & $\mathrm{X}$ & & & & & & & & & \\
\hline P. mesolepta (Ehr.) Sm. & & & & & & & & $\mathrm{X}$ & & & & & \\
\hline Pinnularia sp. Ehr. & & & & $\mathrm{X}$ & & & & $\mathrm{X}$ & & & & & \\
\hline Rhizosolenia sp. Brightwell & & & & $\mathrm{X}$ & & & & & & & & & \\
\hline Surirella $\mathrm{cf}$. linearis Sm. & & & & & & & & $\mathrm{X}$ & & & & & \\
\hline S. guatimalensis Ehr. & & & & $\mathrm{X}$ & & & & & & & & & \\
\hline Surirella spp. (2) Tur. & & & & $\mathrm{X}$ & & & & & & & & & \\
\hline S. tenera Greg. var. nervosa Schim. & & & & & & & & $\mathrm{X}$ & & & & & \\
\hline Synedra goulardii $\mathrm{Br}^{\prime} \mathrm{eb}$. & & & & $\mathrm{X}$ & & & & & & & & & \\
\hline Synedra spp. (2) Ehr. & & & & $\mathrm{X}$ & & & & & & & & & \\
\hline Synedra ulna (Nitzsch) Ehr. & & & & $\mathrm{X}$ & & & & & & & & & \\
\hline Thallassiosira weisflogii (Grun.) Fryx. \& Has. & & $\mathrm{X}$ & & & & & & $\mathrm{X}$ & & & & & \\
\hline Urosolenia eriensis (H. L. Sm.) Round \& Craw. & & & & & & & & $\mathrm{X}$ & & & & & \\
\hline U. eriensis var. morsa (West \& G. S. West) & & & & & & & & $\mathrm{X}$ & & & & & \\
\hline U. longiseta (Zach.) Round \& Craw. & & $\mathrm{X}$ & & & & & & $\mathrm{X}$ & & & & & \\
\hline \multicolumn{14}{|l|}{ Zygnemaphyceae } \\
\hline Closterium acutum var. variable Bréb. & & & & & & & & $\mathrm{X}$ & & & & & \\
\hline C. kutzingii Bre'b & & & $\mathrm{X}$ & & & & & & & & & & \\
\hline C. nordstedtii Chod. & & & & & & & & $\mathrm{X}$ & & & & & \\
\hline C. setaceum Ehr. & & & $\mathrm{X}$ & & & & & $\mathrm{X}$ & & & & & \\
\hline Closterium spp. (7) Nitz. ex Ral. & & $\mathrm{X}$ & $\mathrm{X}$ & & & & & & & $\mathrm{X}$ & & & \\
\hline \multicolumn{14}{|l|}{ C. toxon W. West. } \\
\hline Cosmarium abreviatum Racib. & & & & & & & & $\mathrm{X}$ & & & & & \\
\hline C. Corda ex Ral. & & & $\mathrm{X}$ & & & & & & & & & & \\
\hline C. ornatum var. ornatum Ralfs ex Ralfs & & & $\mathrm{X}$ & & & & & & & & & & \\
\hline Cosmarium spp. (5) Cor. ex Ral. & & $\mathrm{X}$ & $\mathrm{X}$ & & & & & & & & & & \\
\hline Desmidium aptogonum Bréb. \& Goder & & & $\mathrm{X}$ & & & & & & & & & & \\
\hline D. baileyi var. baileyi Nordst. & $\mathrm{X}$ & & $\mathrm{X}$ & & & & & & & & & & \\
\hline D. cylindricum Grev. & $\mathrm{X}$ & & $\mathrm{X}$ & & & & & & & & & & \\
\hline
\end{tabular}


Quadro 1. Cont.

\begin{tabular}{|c|c|c|c|c|c|c|c|c|c|c|c|c|c|}
\hline \multirow{2}{*}{$\begin{array}{l}\text { Bacias } \\
\text { Táxons }\end{array}$} & \multicolumn{7}{|c|}{ Paraguai } & \multicolumn{6}{|c|}{ Paraná } \\
\hline & 1 & 2 & 3 & 4 & 5 & 6 & 7 & 8 & 9 & 10 & 11 & 12 & 13 \\
\hline E. brasiliense Bor. & & & $\mathrm{X}$ & & & & & & & & & & \\
\hline Euastrum spp. (2) Ehr. ex Ral. & & & $\mathrm{X}$ & & & & & & & & & & \\
\hline E. cf. pseudornans Forst. & & & & & & & & $\mathrm{X}$ & & & & & \\
\hline Hyalotheca mucosa (Mert.) Ehr. ex Ral. & $\mathrm{X}$ & & $\mathrm{X}$ & & & & & & & & & & \\
\hline Hyalotheca sp. Ehr. ex Ral. & & & $\mathrm{X}$ & & & & & & & & & & \\
\hline Mougeotia furcata Agard. ex Ral. & & & $\mathrm{X}$ & & & & & & & & & & \\
\hline Mougeotia spp. (3) Agard. & & & $\mathrm{X}$ & & & & & & & $\mathrm{X}$ & & & \\
\hline Sphaerozosma laeve (Nordst.) Thom. & $\mathrm{X}$ & & $\mathrm{X}$ & & & & & & & & & & \\
\hline Sphaerozosma sp. Cor. ex ex Ralf. & & & $\mathrm{X}$ & & & & & & & & & & \\
\hline Spondyloslum sp. Breb. in Dict. & & & & & & & & & & $\mathrm{X}$ & & & \\
\hline S. boergesenii (Boerg.) Racib. & & & $\mathrm{X}$ & & & & & & & & & & \\
\hline S. leptacanthum Nordst. & & & & & & & & $\mathrm{X}$ & & & & & \\
\hline S. leptocladum Nordst. & & & $\mathrm{X}$ & & & & & & & & & & \\
\hline S. quadrangulare var. longispina Borg. & & & $\mathrm{X}$ & & & & & & & & & & \\
\hline S. rotula Nordst. & & & & & & & & $\mathrm{X}$ & & & & & \\
\hline Staurastrum spp. (6) Mey. ex Ralf. & & $\mathrm{X}$ & $\mathrm{X}$ & & & & & & & $\mathrm{X}$ & & & \\
\hline S. tringularis Mey. & & $\mathrm{X}$ & & & & & & & & & & & \\
\hline S. cuspidatus (Br'eb.) Teil. & & & $\mathrm{X}$ & & & & & & & & & & \\
\hline S. extensus (And.) Teil. & & & & & & & & $\mathrm{X}$ & & & & & \\
\hline Staurodesmus spp. (5) Teil. & & $\mathrm{X}$ & $\mathrm{X}$ & & & & & & & $\mathrm{X}$ & & & \\
\hline S. triangularis (Lagerh.) Teil. & & & & & & & & $\mathrm{X}$ & & & & & \\
\hline Xanthidium spp. (2) Ehr. ex Ralf. & & $\mathrm{X}$ & $\mathrm{X}$ & & & & & & & & & & \\
\hline Zygnematales (1) & & & & & & & & & & $\mathrm{X}$ & & & \\
\hline Centritractus belenophorus Lemm & & & & & & & & $\mathrm{X}$ & & & & & \\
\hline Chlamydomonas sp. Ehr. & & & & & & & & & & $\mathrm{X}$ & & & \\
\hline Chromulina sp. Cienkow. & & & & & & & & $\mathrm{X}$ & & & & & \\
\hline D. divergens Imhof & & $\mathrm{X}$ & & $\mathrm{X}$ & & & & $\mathrm{X}$ & & & & & \\
\hline D. sertularia Ehr. & & $\mathrm{X}$ & & $\mathrm{X}$ & & & & & & & & & \\
\hline Dinobryon spp. (2) Ehr. & & & & & & & & $\mathrm{X}$ & & $\mathrm{X}$ & & & \\
\hline Kephyrion spp. (2) Pasch. & & & & & & & & $\mathrm{X}$ & & & & & \\
\hline Mallomonas spp. (4) Pert. & & $\mathrm{X}$ & & $\mathrm{X}$ & & $\mathrm{X}$ & & & & $\mathrm{X}$ & & & \\
\hline Synura spp. (2) Ehr. & & & & $\mathrm{X}$ & & & & & & & & & \\
\hline Cryptophyceae & & & & & & & & & & & & & \\
\hline Chroomonas acuta Uterm. & & & & & & & & $\mathrm{X}$ & & & & & \\
\hline C. nordstedtii Hansg. & & & & & & & & $\mathrm{X}$ & & & & & \\
\hline Chroomonas spp. (3) Hansg. & & $\mathrm{X}$ & & & & & & & & $\mathrm{X}$ & & & \\
\hline C. brasiliensis Bicud. \& Bicud. & & $\mathrm{X}$ & & $\mathrm{X}$ & & & & $\mathrm{X}$ & & & & & \\
\hline C. curvata Ehr. Emend. Pen. & & & & & & & & $\mathrm{X}$ & & & & & \\
\hline C. marssonii Skuja & & & & $\mathrm{X}$ & & & & & & & & & \\
\hline C. ovata Ehr. & & & & $\mathrm{X}$ & & & & & & & & & \\
\hline Cryptomonas spp. (3) Ehr. & & $\mathrm{X}$ & & $\mathrm{X}$ & & & & & & & & & \\
\hline Rhodomonas lacustris Pasch. \& Rut. & & & & & & & & $\mathrm{X}$ & & & & & \\
\hline R. minuta Skuja & & & & $\mathrm{X}$ & & & & & & & & & \\
\hline Xanthophyceae & & & & & & & & & & & & & \\
\hline Goniochloris fallax Fott & & & & & & & & $\mathrm{X}$ & & & & & \\
\hline G. tripus Pasch. & & & & $\mathrm{X}$ & & & & & & & & & \\
\hline Isthmochloron gracile (Reins.) Sku. & & $\mathrm{X}$ & & & & & & $\mathrm{X}$ & & & & & \\
\hline P. lobulatumn (Näg.) Chodat & & & & $\mathrm{X}$ & & & & & & & & & \\
\hline Tetraedriella jovetii (Bourr.) Bourr. & & & & $\mathrm{X}$ & & & & & & & & & \\
\hline T. regularis (Kütz.) Fott & & & & & & & & $\mathrm{X}$ & & & & & \\
\hline Tetraplektron spp. (3) Fott & & & & & & $\mathrm{X}$ & & & & & & & \\
\hline T. torsum (Skuja) Dedus. Sceg. & & & & $\mathrm{X}$ & & & & & & & & & \\
\hline Dinophyceae & & & & & & & & & & & & & \\
\hline Peridinium spp. (8) Ehr. & & $\mathrm{X}$ & & $\mathrm{X}$ & & & & $\mathrm{X}$ & & & & & \\
\hline Glaucocystis sp. Itzig. & & $\mathrm{X}$ & & & & & & & & & & & \\
\hline Raphydophyceae & & & & & & & & & & & & & \\
\hline Gonyostomum sp. Dies. & & $\mathrm{X}$ & & & & & & & & & & & \\
\hline
\end{tabular}


A tabela 1 lista as localidades de amostragens com suas respectivas coordenadas geográficas e a figura 1 mostra as áreas de amostragens para organismos fitoplanctônicos e suas respectivas publicações. Dos 13 trabalhos que abordaram populações fitoplanctônicas e que tiveram ou citaram o estado do Mato Grosso do Sul como local de estudo, sete foram na bacia do Alto Paraguai e seis na bacia do Paraná. Entretanto, destes trabalhos oito foram publicados em revistas científicas, os demais foram resumos de congressos, dissertações de mestrado; destes apenas três foram realizados por pesquisadores vinculados a instituições sul-mato-grossenses (Oliveira \& Calheiros 2000, Malone et al. 2008 e Vieira et al. 2009). Os registros das espécies das comunidades fitoplanctônicas foram feitos em sua grande maioria por estudos ecológicos (97\%) e os estudos de taxonomia sensu stricto foram três (Malone et al. 2008, Santos \& Sant'Anna, 2010, Tremarin et al. 2014) com registro de 3\% das espécies listadas para o estado. Segundo Nabout et al. (2015) o estado de Mato Grosso do Sul esta entre os estados com menor produção intelectual sobre fitoplâncton no Brasil.

\section{Grupos de Pesquisa no estado de Mato Grosso do Sul}

Não há no estado de Mato Grosso do Sul taxonomistas em nenhum grupo de algas fitoplanctônicas. Entretanto, os grupos de pesquisas em limnologia vinculados ao Programa de Pós-Graduação em Tecnologias Ambientais da Universidade Federal de Mato Grosso do Sul tem produzido dissertações abordando diferentes aspectos do fitoplâncton. Estes estudos abordam principalmente a ecologia de fitoplâncton, produção de cianobactérias para extração e identificação de cianotoxinas e utilização de algas para produção de biodiesel (UFMS 2012). Pesquisadores que orientam em limnologia, em instituições sul-mato- grossenses, que abordam a ecologia de fitoplâncton são Prof. Dr. Kennedy Roche, UFMS, Campo Grande; Prof. Dr. William Marcos da Silva, UFMS, Campus Pantanal, Corumbá; Prof. Dr. Ricardo Henrique Gentil Pereira, UFMS, Campus Aquidauana, Dra. Marcia Divina de Oliveira, Embrapa/Pantanal, Corumbá.

Pesquisadores de instituições não sul-mato-grossenses, mas que possuem sítios de estudos no Mato Grosso do Sul: Profa. Dra. Sueli Train, Profa. Dra. Luzia Cleide Rodrigues e Profa. Dra. Susicley Jati, do Núcleo de Pesquisas em Limnologia Ictiologia e Aquicultura (NUPELIA), Maringá Paraná; Profa. Dra. Célia Leite Sant'Anna, Instituto de Botânica de São Paulo, Universidade de São Paulo; Profa. Dra. Maria José Neto do UFMS, Campus de Tres lagoas.

\section{Lacunas do conhecimento}

As lacunas de conhecimento para os organismos fitoplanctônicos no estado são muito grandes, tanto para a taxonomia quanto para ecologia. A principal lacuna para o conhecimento dos organismos fitoplanctônicos é a falta de especialistas nos programas de Pós-Graduação, que reflete na baixa produção científica sobre o tema, e na falta de amostragens nos corpos de água no interior do estado do Mato Grosso do Sul. O presente inventário mostrou que um grande número dos organismos foi registrado até ao nível de gênero apenas, logo trabalhos de aprofundamento taxonômico fazem-se necessários.

\section{Principais acervos}

Os acervos de organismos fitoplanctônicos do estado estão em coleções preservadas em formaldeído $4 \%$, em lugol-acético e em solução Transeau, todas depositadas nos seguintes laboratórios: Laboratório de Microbiologia

Tabela 1. Lista das localidades e coordenadas geográficas, onde * são locais sem coordenadas e ** são locais com vários pontos não especificados e respectivas referências.

\begin{tabular}{|c|c|c|c|}
\hline Localidade & Latitude (S) & Longitude (W) & Referências \\
\hline Corumbá* & & & Martins (1980) \\
\hline Corumbá & $18^{\circ} 59^{\prime}$ & $57^{\circ} 34^{\prime}$ & Oliveira \& Calheiros (2000) \\
\hline Lagoa do Castelo & $18^{\circ} 34^{\prime}$ & $57^{\circ} 38^{\prime}$ & Oliveira \& Calheiros (2000) \\
\hline Corumbá & $18^{\circ} 59^{\prime}$ & $57^{\circ} 42^{\prime}$ & Silva et al. (2002) \\
\hline Rio Paraguai - Carceres ate foz rio Apa** & $16^{\circ} 03^{\prime}-22^{\circ} 00^{\prime}$ & $57^{\circ} 13^{\prime}-58^{\circ} 23^{\prime}$ & Domitrovic (2002) \\
\hline Pantanal da Nhecolândia-Salina da Ponta & $18^{\circ} 59^{\prime} 00^{\prime}$ & $56^{\circ} 39^{\prime} 35^{\prime \prime}$ & Malone et al. (2008) \\
\hline Pantanal da Nhecolândia-Salina da Reserva & $18^{\circ} 57^{\prime} 42^{\prime \prime}$ & $56^{\circ} 37^{\prime} 26^{\prime \prime}$ & Malone et al. (2008) \\
\hline Represa Córrego Alegre-Rio Miranda** & $19^{\circ} 15^{\prime}-22^{\circ} 00^{\prime}$ & $54^{\circ} 15^{\prime}-57^{\circ} 30^{\prime}$ & Vieira et al. (2009) \\
\hline Pantanal da Nhecolâdia - Salina Campo Dora & $18^{\circ} 58^{\prime} 02^{\prime \prime}$ & $56^{\circ} 38^{\prime} 59^{\prime \prime}$ & Santos \& Sant' Ana (2010) \\
\hline Pantanal da Nhecolâdia - Salina do meio & $18^{\circ} 58^{\prime} 29^{\prime \prime}$ & $56^{\circ} 38^{\prime} 47^{\prime \prime}$ & Santos \& Sant'Ana (2010) \\
\hline Pantanal da Nhecolâdia - Baía da sede Nhuminrim & $18^{\circ} 59^{\prime} 37^{\prime \prime}$ & $56^{\circ} 37^{\prime} 14^{\prime \prime}$ & Santos \& Sant ${ }^{\prime}$ Ana (2010) \\
\hline Represa Lago do Amor & $20^{\circ} 30^{\prime} 12^{\prime \prime}$ & $54^{\circ} 37^{\prime} 00^{\prime \prime}$ & Ferro et al. (2008) \\
\hline Rio Baía & $22^{\circ} 43^{\prime}$ & $54^{\circ} 17^{\prime}$ & Train \& Rodrigues (1998) \\
\hline Represa Lago do Amor & $20^{\circ} 30^{\prime} 12^{\prime \prime}$ & $54^{\circ} 37^{\prime} 00^{\prime \prime}$ & Lopes (2009) \\
\hline Rio Ivinhema-Rio Paraná** & $22^{\circ} 45^{\prime}-22^{\circ} 50^{\prime}$ & $53^{\circ} 22^{\prime}-53^{\circ} 32^{\prime}$ & Train et al. (2009) \\
\hline Represa Lago do Amor & $20^{\circ} 30^{\prime}$ & $54^{\circ} 37^{\prime} 00^{\prime \prime}$ & Silva (2012) \\
\hline
\end{tabular}




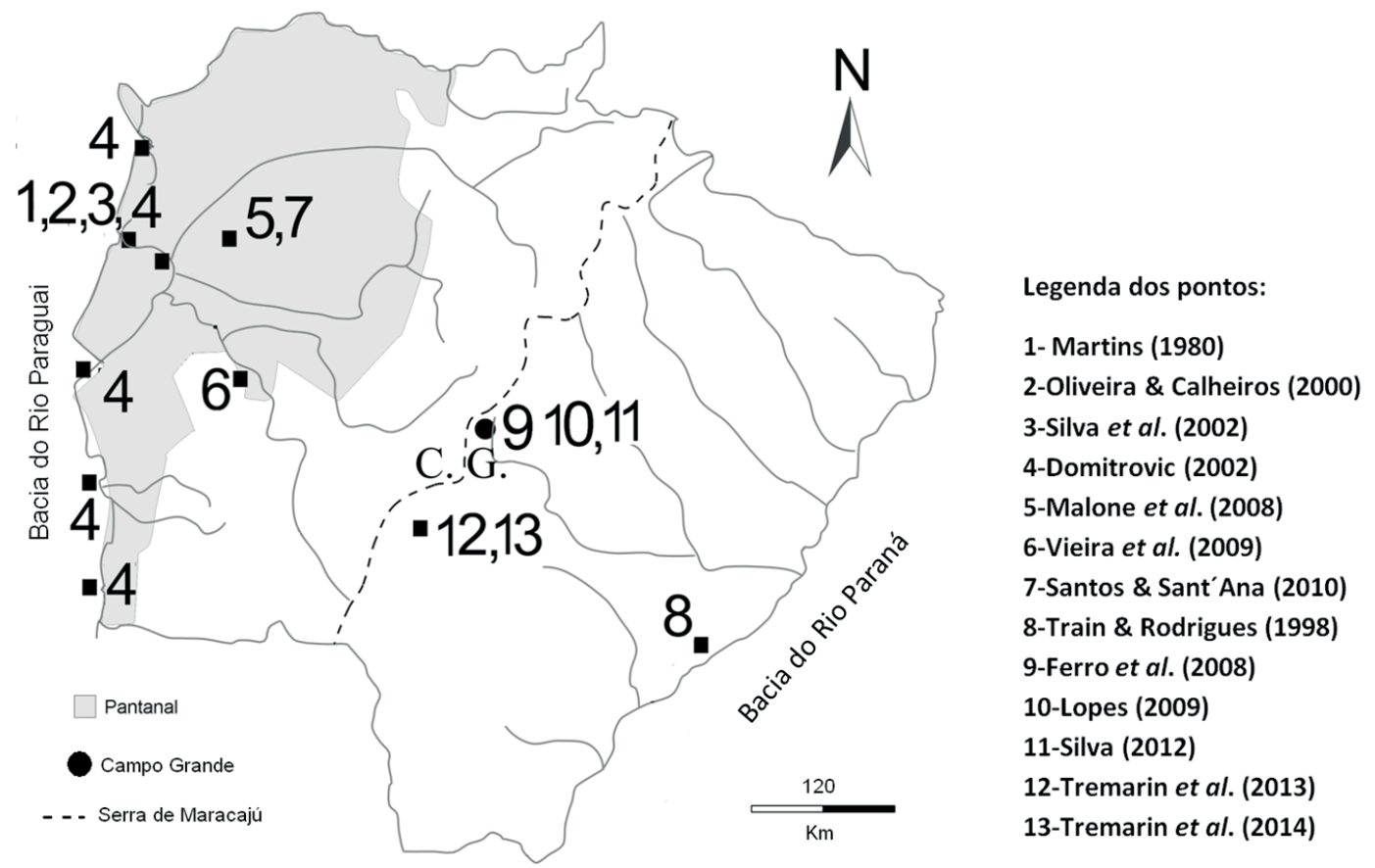

Fig. 1. Distribuição dos locais de amostragens de fitoplâncton no estado de Mato Grosso do Sul com seus respectivos autores.

Ambiental da Universidade Federal de Mato Grosso do Sul de Campo Grande, Laboratório de Ecologia da Universidade Federal de Mato Grosso do Sul, Campus Corumbá e Laboratório de Biologia da Universidade Federal de Mato Grosso do Sul Campus de Aquidauana, e no herbário da Embrapa CPAP/Corumbá. Existem, também, amostras no Instituto do Meio Ambiente do estado do Mato Grosso do Sul, coletadas em monitoramento ambiental da região de Ivinhema. Outros acervos fora do estado que possuem amostras de fitoplâncton coletadas em corpos de água do Mato Grosso do Sul estão depositados no NUPELIA, UEM, Maringá, Paraná; no Museu do Plâncton da Universidade Federal de São Carlos, São Paulo e no Instituto de Botânica da Universidade de São Paulo, São Paulo.

Perspectivas de pesquisa de organismos fitoplanctônicos para os próximos 10 anos

As perspectivas de pesquisas no estado de Mato Grosso do Sul para os organismos fitoplanctônicos são muito grandes. Existe uma demanda a ser suprida imediatamente nos órgãos de fiscalização ambiental do estado, assim como nas empresas de produção de água, as quais têm a obrigação legal de fazer e cobrar o biomonitoramento de empreendimentos que utilizam os recursos hídricos. Entretanto, o subsídio ao monitoramento e à fiscalização é dependente da formação e treinamento de recursos humanos. Esta formação irá gerar conhecimento e pesquisas nos corpos de água do estado para estabelecer as particularidades regionais, tanto de composição taxonômica quanto de ecologia e aspectos fisiológicos, tais como produção de toxinas. Para um aumento imediato do conhecimento taxonômico dos organismos seriam necessários convênios com instituições de pesquisa com notório reconhecimento na área, para treinamento de alunos de iniciação científica e de Pós-Graduação de programas de instituições de ensino e pesquisa do estado de Mato Grosso do Sul. Uma estratégia válida seria atrair pesquisadores para o estado por meio de bolsa de desenvolvimento regional (DCR) ou de professor visitante. Ambos para atuar na pesquisa e formação de recursos humanos em Programas de Pós-Graduação e com isso criar demandas para os postos de pesquisadores efetivos no estado.

Segundo Bicudo et al.(1996) e Silva \& MatsumuraTundisi (2011) o gargalo na formação de taxonomistas está na falta de atratividade da área na atual grade dos cursos de Ciências Biológicas, que é o principal fornecedor de recursos humanos para taxonomia. Portanto, a criação e fomento de grupos de pesquisa que abordem a taxonomia e ecologia de algas planctônicas são necessários para atrair estudantes de iniciação e de pós-graduação, além de ampliar e melhorar a qualidade de monitoramento ambiental que é a base do uso sustentável dos recursos hídricos.

\section{AGRADECIMENTOS}

A Fundação de Apoio ao Desenvolvimento do Ensino, Ciências e Tecnologia do Estado de Mato Grosso do Sul e a Superintendência de Ciências e Tecnologia do Estado de Mato Grosso do Sul pelo convite de participação neste volume especial e o suporte financeiro para sua publicação. 


\section{REFERÊNCIAS}

Agostinho, A.A., Thomaz, S.M. \& Gomes, L.C. 2005. Conservação da biodiversidade em águas continentais do Brasil. Megadiversidade 1(1): $70-78$

Barbosa, F.A.R., Bicudo, C.M., \& Huszar, V.L.1995 Phytoplankton studies in Brazil: community structure, variation and diversityJ.G. Tundisi, C.M. Bicudo, T.M. Tundisi (Eds.), Limnology in Brazil, Brazilian Academy of Sciences, Rio de Janeiro pp. 19-36.

Bicudo, C.E.M. Bicudo, D.C. \& Giani, A. 1996. Towards assaying biodiversity in freshwateralgae. In Biodiversity in Brazil, a first approach (C. E. M. Bicudo, \& N. A. Menezes, eds.). São Paulo: Conselho Nacional de Desenvolvimento Científico e Tecnológico. p. 5-16.

Bicudo, C.E.M. \& Menezes, M. 2010. Algas do Brasil. In Forzza, R.C.; Leitman, P.M.; Costa, A.; Carvalho-Júnior, A.A.; Peixoto, A.L.; Walter, B.M.T.; Bicudo, C.; Zappi, D.; Costa, D.P.; Lleras, E.; Martinelli, G.; Lima, H.C.; Prado, J.; Stehmann, J.R.; Baumgratz, J.F.A.; Pirani, J.R.; Sylvestre, L.S.; Maia, L.C.; Lohmann, L.G.; Paganucci, L.; Silveira, M.; Nadruz, M.; Mamede, M.C.H.; Bastos, M.N.C.; Morim, M.P.; Barbosa, M.R.; Menezes, M.; Hopkins, M.; Secco, R.; Cavalcanti, T. \& Souza, V.C. (Eds.). Catálogo de Plantas e Fungos do Brasil. v. 1. Rio de Janeiro p 49-60.

Branco, S.M. 1986. Hidrobiologia aplicada à Engenharia Sanitária. Companhia de Tecnologia de Saneamento Ambiental/Associação dos Funcionários da Companhia de Tecnologia de Saneamento Ambiental, São Paulo. p. 616.

Cavalier-Smith, T . 2009.Kingdoms Protozoa and Chromista and the eozoan root of the eukaryotic tree. Biology Letters Disponível em: http://rsbl.royalsocietypublishing.org/content/early/2009/12/17/ rsbl.2009.0948.full.html\#ref-list-1. Acessado em 22.08.2012.

Cavalier-Smith, T. 2004. Only six kingdoms of life. Proceedings of the Royal Society B: Biological Sciences. 271, 1251-1262.

Chretiennot-Dinet, M.J. \& Ricard, M. 1991. Marine phytoplankton: how many species in the world? Journal of Plankton. Res. 13:1093-1099.

Cybis, L.F., Bendati, M.M., Maizonave, C.R.M., Werner, V.R. \& Domingues, C.D. 2006. Manual para estudo de cianobactérias planctônicas em mananciais de abastecimento público caso da represa Lomba do Sabão e Lago Guaíba, Porto Alegre, Rio Grande do Sul. Rio de Janeiro: Associação Brasileira de Engenharia Sanitária e Ambiental. 64p.

Domitrovic, Y. 2002. Structure and variation of the Paraguay River phytoplankton in twoperiods of its hydrological cycle. Hydrobiologia 472:177-196.

Espíndola, E.G., Matsumura-Tundisi, T. \& Moreno, I.D. 1996. Estrutura da comunidade fitoplanctônica da lagoa Albuquerque (Pantanal Mato-grossense), Mato Grosso do Sul, Brasil. Acta Limnologica Brasiliensia 8:13-27

Ferro, P. O. M. P. L. C., Silva, W. M., Rochie, K. F. \& LOPES, I. R. 2008. Composição das populações fitoplanctônicas nos meses dedezembro/2006 e junho/2007 em um lago urbano, represa lago do amor (Campo Grande, MS). Resumos do XII Congresso Brasileiro de Ficologia, Águas Claras, Brasília, Distrito Federal.p. 47

Guiry, M.D. 2012. How many species of algae are there? Phycological Society of America. Kasting, F.K. \& Siefert, J.L. 2002. Life and the Evolution of Earth's Atmosphere. Science 296, 1066.

Kasting, J.F. \& Seifert, J.L. 2002. Life and the evolution of Earth's atmosphere. Science 0296, 1066-8.

Kruk, C., Segura, A. M., Peeters, E.T.H.M. ,M. Huszar, V. L., Costa, L. S., Kosten, S., Lacerot, G. \& Scheffer, M. 2012. Phytoplankton species predictability increases towards warmer regions. Association for the Sciences of Limnology and Oceanography 57(4), 1126-1135.

Lopes, I.R. 2009. Estudo das assembléias fitoplanctônicas de uma pequena represa urbana eutrofizada, o Lago do Amor, com ênfase em interações com macrófitas aquáticas e a sazonalidade. Dissertação f.. Universidade Federal de Mato Grosso do Sul. Disponível em: https:// sistemas.ufms.br/sigpos/portal/trabalhos/download/.../cursoId:3. Acessado em 22.08.2012.

Malone, C.F.S, Santos, K.R.S, Neto, M.J. \& Sakamoto, A.Y. 2008. Gêneros de algas no plâncton de lagoas salinas situadas na fazenda
Nhumirim, Pantanal da Nhecolândia, MS. Revista Brasileira de Biociências 5:588-590.

Margalef, R. 1983. Limnologia. Omega, Barcelona.1.010 p.

Martins, D.V.1980. Contribuição à ficologia da Amazônia. Desmídoflorula dos lagos Cristalino e São Sebastião, Estado do Amazonas: Gêneros filamentosos. Acta Amazonica.10(4):725-745.

Mato Grosso do Sul. 1990. Secretaria de Planejamento e Coordenação Geral. Atlas multirreferencial do Estado do Mato Grosso do Sul. Campo Grande. Governo Estadual, Instituto Brasileiro De Geografia E Estatística.

Nabout, J.C., Carneiro, F.M., Borges, P.P., Machado, K.B., \& Huszar, V.L.M. 2015. Brazilian scientific production on phytoplankton studies: national determinants and international comparisons. Brazilian Journal of Biology, 75(1): 216-223.

Oliveira. M.D. \& Calheiros. D. F. 2000. Flood pulse influence on phytoplankton communities of thesouth Pantanal floodplain, Brazil. Hydrobiologia 427: 101-112.

Padisák J., Luciane, E., Crossetti, O. \& Naselli-Flores, L. 2009.Use and misuse in the application of the phytoplankton functional classification: a critical review with updates. Hydrobiologia 621:1-19.

Reviers, B. 2006. Biologia e filogenia das algas. Artmed, Porto Alegre. $280 \mathrm{p}$.

Reynolds, C. S., V. Huszar, C. Kruk, L. Naselli-Flores \& S.Melo. 2002. Towards a functional classification of thefreshwater phytoplankton. Journal Plankton Research 24: 417-428.

Rocha, O. 2006. Organismos de água doce. In Avaliação do Estado do Conhecimento da Diversidade Biológica Brasileira (T. M. Lewinsohn, Org.). Ministério do Meio Ambiente, Brasilia. v.2. Disponível em: http://www.mma.gov.br/estruturas/chm/_arquivos/Aval_Conhec_ Cap5.pdf. Acessado em 08.09.2015

Santos, K.R.S. \& Sant'Anna, C.L. 2010. Cianobactérias de diferentes tipos de lagoas ("salina", "salitrada" e "baía") representativas do Pantanal da Nhecolândia, MS,Brasil. Revista Brasileira de Botânica 33(1): 61-83.

Silva, E.L.V, Oliveira, M. D. \& Ishii, I.I. 2000. Estrutura da comunidade fitoplanctônica no rio Paraguai e canal do tamengo, Pantanal, MS. In Anais do III Simpósio sobre Recursos Naturais e Sócio-econômicos do Pantanal (E.K. Rezende, A.O. Pellegrin, J.A.C. Filho \& L.A. Pellegrin, coords.). Embrapa Pantanal, Corumbá. Publicação eletrônica. Disponível em: http://www.cpap.embrapa.br/publicacoes/ simposios.html. Acessado em: 22.08.2012.

Silva, S.V. 2012. Identificação e avaliação da toxicidade de cianobactérias do lago do amor e da represa de abastecimento Guariroba. Tese 69 f., Universidade Federal de Mato Grosso do Sul.

Silva, W.M. \& Matsumura-Tundisi, T. 2011. Checklist dos Copepoda Cyclopoida de vida livre de água doce do Estado de São Paulo, Brasil. Biota Neotropica 11(1a): 5-11.

Train, S. \& Rodrigues, LC. 1998. Temporal fluctuations of the phytoplankton community of the Baía River, in the upper Paraná River floodplain, Mato Grosso do Sul, Brazil. Hydrobiologia 361: 125-134.

Tremarin, P.I., Freire, E. G., Bertolli, L. M, \& Ludwig. T. A. V. 2009. Catálogo das diatomáceas (Ochrophyta-Diatomeae) continentais do estado do Paraná. Iheringia. Série Botânica 64(2):79-107.

Tremarin, P.I., Ludwig, T.A.V. \& Torgan, L.C. 2013. Morphological variation and distribution of the freshwater diatom Aulacoseira ambigua (Grunow) Simonsen in Brazilian continental environments. Iheringia, Série Botanica 68: 139-157.

Tremarin, P.I., Ludwig, T.A.V. \& Carvalho, L.T. 2014. Aulacoseira veraluciae sp. nov. (Coscinodiscophyceae, Aulacoseiraceae): a common freshwater diatom from Brazil. Phytotaxa 184(4): 208-222.

Tundisi, JG. \& Matsumura-Tundisi, T. 2008. Limnologia. Oficina de Textos. $632 \mathrm{p}$.

UFMS. 2012. Universidade Federal de Mato Grosso do Sul, Pró-Reitoria de Pesquisa e Pós-Graduação. Disponível em: http://www.propp. ufms.br/. Acessado em 10.09.2012.

Vieira, B.H., Pereira, R.H.G. \& Derbócio, M. 2009. Análise qualitativa da comunidade fitoplanctônica de um ecossistema aquático utilizado para o cultivo de peixes em tanque-rede, Pantanal de Miranda, MS. Boletim do Instituto de Pesca 35(4):567-576. 